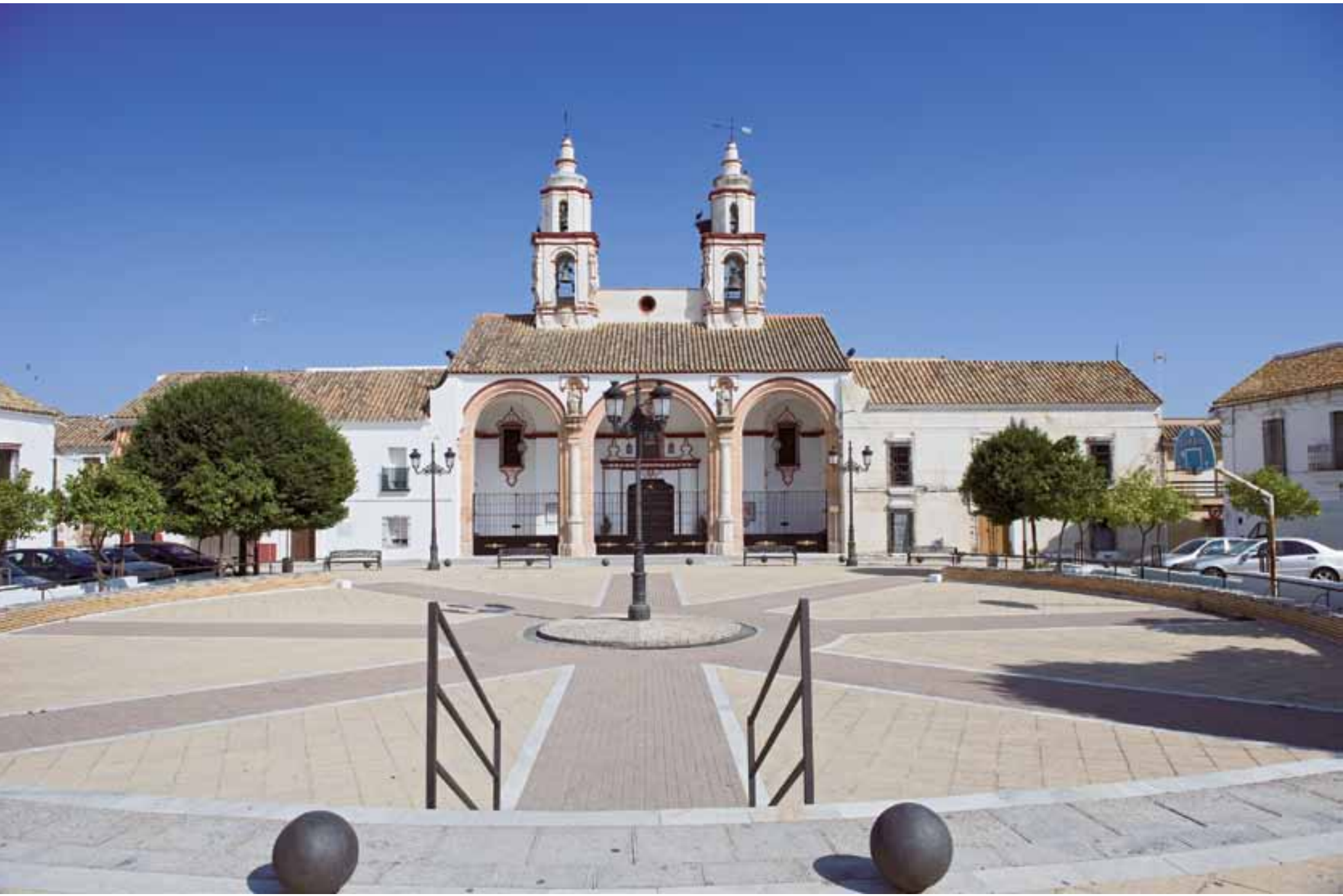

Plaza de la Iglesia de la Purísima Concepción en La Carlota. Foto: Juan Carlos Cazalla, IAPH 


\section{Equilibrio territorial: las nuevas poblaciones de Andalucía del siglo XVIII}

Jordi Oliveras Samitier, Dpto. de Composición Arquitectónica, U. de Barcelona
Quien tenga curiosidad y pueda caminar, ir en bicicleta o en coche, por la zona de la campiña entre Córdoba y Écija y entre Écija y Carmona -o también sobrevolarla aunque sea virtualmente a través de Google Earth o de la magnífica cartografía del Instituto de Cartografía de Andalucia accesible en red-, puede reseguir las líneas maestras de la colonización que hace casi 250 años se llevó a cabo. La Carlota era la capital de esta "subdelegación" de Nuevas Poblaciones. Otras feligresias o ciudades mayores fundadas en la época fueron La Luisiana, Fuente Palmera, y San Sebastián de los Ballesteros y, cerca, otras aldeas o pueblos menores dependientes de éstas. Así satélites de La Carlota fueron: Fuencubierta, La Chica Carlota, Garabato, Pinedas y Quintana; satélites de La Luisiana fueron Campillo, Cañada Rosal y Los Motillos; y aldeas de Fuente Palmera fueron: Ochavillo del Río, Herrería, La Ventilla, Villalón, La Peñalosa, Fuente Carreteros y Silillos.

La parcelación del campo en la porción norte de la carretera (hoy A-364), cerca de La Luisiana, es distinta a la del resto del campo andaluz aún después de 250 años del inicio de la colonización. Una serie de parcelas profundas dan fachada al antiguo camino de Cádiz a Madrid. Entre La Luisiana y Cañada Rosal, y más concretamente entre Campillo y Cañada Rosal, la carretera (SE-9104) es la "línea maestra" de la parcelación agrícola de esta porción de terrenos que ocupan parcialmente lo que los hombres del siglo XVIII Ilamaban el desierto de la Monclova y que convirtieron en campos de cultivo agricola, pasto y residencia de colonos. Paralela a la línea maestra, otras líneas, a una distancia entre si de 1000 varas (unos 800 metros), marcan los caminos de acceso a las parcelas de cultivo de 500 varas de ancho (unos 400 metros), por tanto, de una extensión aproximada de 32 ha, equivalentes a las 50 fanegas de tierra que se otorgaban a cada colono. En reconocimientos posteriores a la colonización (por ejemplo, en el de Tomás González de Carvajal de 1796) se da cuenta de que las suertes o parcelas de La Luisiana miden: Ias que dan a la carretera o Camino Real, 42 fanegas; y las demás, 56 fanegas, a menudo subdivididas en dos suertes de 28 cada una.
En La Carlota, si seguimos la N-IVa, que es la ruta que coincide mejor con la antigua carretera de Córdoba a Sevilla, vemos cómo surgen a partir de ella las líneas maestras de la parcelación tiradas a cordel, a una distancia de 1000 varas, iguales a las anteriores. Desde la plaza de la iglesia, en frente de ésta, hacia el sureste, surge una linea que es el llamado camino de La Carlota y que hace de principal por este lado sur del Camino Real. Hacia el noroeste desde la perpendicular a la carretera principal surge otra linea que va hasta Fuencubierta (por la actual Calle Redonda de la Fuente nueva), y que es la maestra a partir de la cual se trazaron otras demarcaciones paralelas para dar lugar a otras parcelas de aproximadamente 50 fanegas.

En Fuente Palmera, si entramos viniendo desde La Carlota, su capital administrativa, pasando por Fuencubierta y La Ventilla (la actual Calle de los Portales), nos lleva a la Plaza Real enfrente de la iglesia. Perpendicular a ella, ya en la plaza, estaba el Camino de Écija (calle Écija), que hacia el sur seria aproximadamente la línea maestra de la demarcación como se dividieron los lotes de cultivo. Hacia el norte la Calle Carlos III y la Carretera de Posadas serian la línea maestra más larga. Cada 800 metros aproximadamente (unas 1000 varas) encontramos una línea de parcelación y después subdivisiones perpendiculares de 400 metros (unas 500 varas). En este caso las parcelas o suertes se subdividieron en dos o más.

Toda esta malla rectangular abarca los términos que se repartian, prescindiendo bastante de los accidentes topográficos, como riachuelos o pequeños montículos, y formando un conjunto fácil de trazar, dividir y controlar. Las parcelas resultantes se concedieron a los colonos por sorteo, de ahi el nombre de "suertes".

En San Sebastián de los Ballesteros la delimitación del término es menos amplia y la línea maestra va adaptándose al camino principal. El terreno había pertenecido a la Compañía de Jesús y, como centro de la población, se aprovechó la iglesia existente y el convento contiguo (igual que se habia hecho en La Peñuela o La Carolina en la Sierra Morena). No en balde, la promulgación del fuero que dio fundamento legal a estas colonizaciones coincidió con la orden de expulsión de la Compañia de Jesús. 


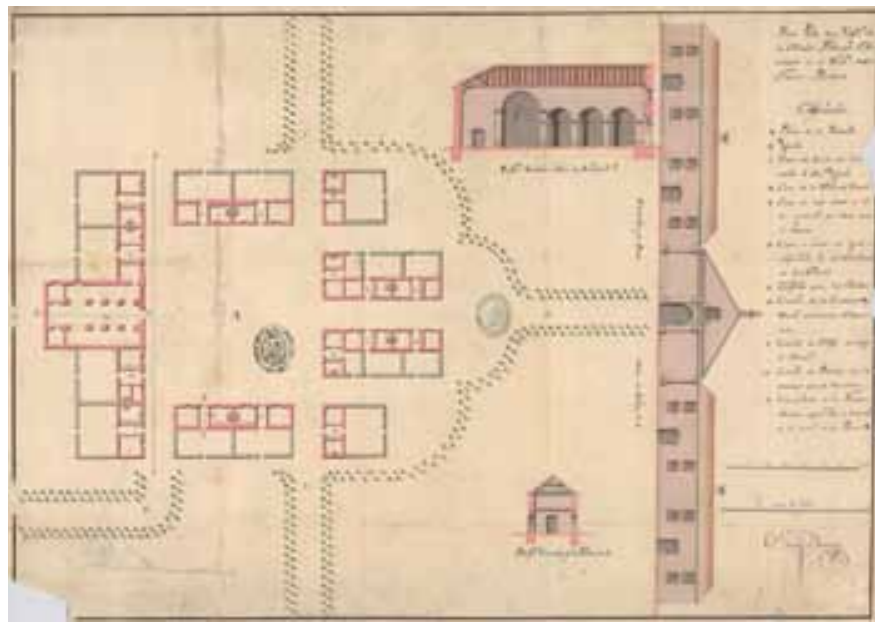

Plano de Vista y Perfil de la Nueva Población ejecutada en el Sitio de Fuente Palmera (Simon Desnaux, 1768?). Fuente: Archivo General Militar de Madrid (IHCM) $\mathrm{SH} . \mathrm{CO}-16 / 6$

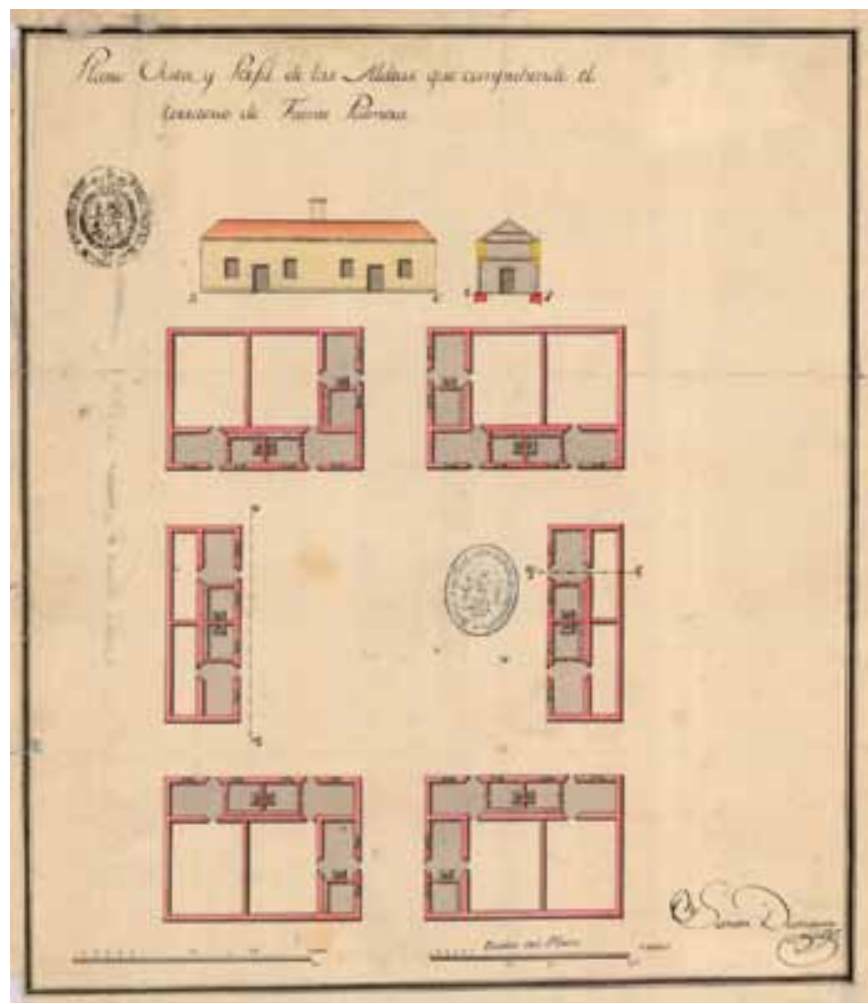

Plano Vista y Perfil de las aldeas que comprende el territorio de Fuente Palmera (Simon Desnaux, 1768?). Fuente: Archivo General Militar de Madrid (IHCM). SH.CO-16/7

La malla parcelaria resultante respondía al deseo de cambiar el estado de la agricultura, tal como se encontraba en la segunda mitad del siglo XVIII. La conciencia de la necesidad de un cambio habia ido en aumento y las decisiones reformadoras y legislativas fueron retrasándose. Pero esta parcelación y colonización llevada a término en Andalucia demuestran el interés de empezar una acción que de forma exploratoria y experimental fuera en la dirección de una reforma más amplia. Las ideas de los reformadores como el Conde de Aranda, Campomanes, Floridablanca, Olavide o Jovellanos, han sido analizadas con detalle por estudiosos que han visto en las distintas medidas propuestas su mayor o menor absolutismo o liberalismo, su conocimiento más preciso de distintas realidades regionales y su oportunidad política de aplicación. Para el caso que nos ocupa las políticas propugnadas por Campomanes y por Olavide resultan de gran trascendencia por ser ellos quienes auspiciaron y dirigieron directamente la experiencia de Sierra Morena y Andalucia. Así las hipótesis en las que se basaban eran primordialmente tres: la desigual distribución de la riqueza agraria, con gran número de pobres; la posibilidad de poner a prueba una reforma persuasiva encabezada por el Estado, sin llegar a coacciones; y que la agricultura con la ganadería debían complementarse, e incluso suplementarse con una industria inicialmente doméstica. Según Olavide una primera fase de reforma podia centrarse en los baldios, que eran muy extensos y en las tierras de propios, en principio destinadas a costear servicios públicos municipales pero mal gestionados. Tanto Campomanes, cuando fue fiscal de Extremadura, como Olavide, asistente de Sevilla, contribuyeron al Expediente de Reforma Agraria proponiendo ideas aplicables como la de distribuir estas tierras inutilizadas en lotes de 50 fanegas para cultivar y dar además un terreno para pasto. Olavide proponia que los pelantrines o pequeños arrendatarios que ya cultivaban hasta 20 fanegas y disponian de yunta pera labrar fueran adjudicatarios de parcelas más extensas donde establecer su unidad de cultivo familiar. Y por otra parte, que los braceros sin tierra tuvieran un lote de dos fanegas en régimen de censo o arrendamiento de un octavo de la producción, que estuviera situado cercano al pueblo para asi poderse desplazar con facilidad. A los agricultores con más posibilidades económicas se les permitiria comprar un lote de hasta 200 fanegas. Los lotes mayores, de hasta 2000 fanegas, serian para fortunas capaces, obligándoles a arrendarlos, así mismo, en lotes de 50 fanegas. Olavide preveia la indivisibilidad de los terrenos para no caer en el minifundio y proponía establecer cotos al abuso de los arrendamientos. Con todas estas medidas se pretendía mejorar notablemente la agricultura y elevar en definitiva el nivel de las rentas inferiores.

Es en este marco de ideas sobre la reforma agricola cuando, en 1768, surgió la oportunidad de extender la legislación del Fuero de las Nuevas Poblaciones de Sierra Morena de 1767 a dos zonas más de Andalucía, en los desiertos de la Parrilla y de la Monclova. El fuero de colonización se promovió también con el fin de establecer poblaciones en zonas despobladas junto al nuevo trazado del Camino Real de Cádiz a Madrid, y así protegerlo. La política del Consejo de Castilla fue sensible a este motivo -dado el bandolerismo que habia en Sierra Morena y también entre Córdoba y Écija en la cuesta del Espino- y así aunarlo al de reformar la agricultura. Por eso poblaciones importantes como La Carlota y La Luisiana fueron realizadas apoyando el camino, como lugares de Postas y servicios para el viajero y como protección del transporte de mercancias favoreciendo así el libre comercio.

Había, sin embargo, otra razón subyacente a la agrícola y a la mercantil, era la de solucionar la despoblación de amplias zonas del territorio y lograr así una mejor distribución de la población. Esta última finalidad coincidia con una nueva conciencia de la necesidad de prevenir y limitar el crecimiento desmesurado de las grandes urbes, por los problemas que se empezaba a comprobar 
que éste ocasionaba. El equipo gobernante habia entendido las consecuencias que podian derivarse de los motines del pan en varias poblaciones y en Madrid particularmente (motin de Esquilache). Pero además, se empezaban a observar los peligros de densidades de población elevadas con resultados de insalubridad y falta de seguridad. Asi Cabarrús, Ponz y Olavide reflejaron en sus escritos e informes estas realidades, y abogaron por su solución con un mayor equilibrio territorial y una limitación del tamaño de las urbes. Es en esta politica de la llustración donde apreciamos un moderno concepto de ordenación equilibrada del territorio, que tanta controversia debería tener en las teorias y las prácticas de Urbanismo de tiempos posteriores.

En este contexto es en el que se puede entender cómo se formalizó la idea de fundar una serie de nuevos pueblos y aldeas de colonización en los que situar la Administración, el pósito, el horno, la herreria, algunos artesanos no labradores, la iglesia, la posada y otros servicios para los campesinos y los viajeros. Fue establecida una jerarquia entre capital de la Delegación de las Nuevas Poblaciones, que fue La Carlota, un segundo rango de feligresías como La Luisiana, Fuente Palmera y San Sebastián de los Ballesteros, y, en tercer nivel, una serie de aldeas dependientes de estas feligresias. Se trataba, por tanto, de un sistema estructurado para no doblar esfuerzos de Administración y servicios, y ofrecer centros de población en aldeas para las tierras inmediatas y para las casas de los alrededores, de manera que la población estuviera mejor diseminada en el territorio. Para que la implantación tuviera éxito, las distancias entre núcleos de población estuvieron bien estudiadas. En teoría, el fuero establecía esta jerarquía y agrupación de pueblos ("...cada tres, cuatro o cinco formen una feligresía o concejo..."), así como la distancia de un cuarto de legua o medio cuarto entre poblaciones (lo que sería aproximadamente entre 0,750 y 1,5 kilómetros). Efectivamente, si observamos la situación de las aldeas y feligresías vemos un reparto homogéneamente calculado, formando nudos de una red de caminos. Casi todos los pueblos están a una distancia equivalente al pueblo vecino. Así encontramos las aldeas de Fuente Palmera muy equidistantemente situadas entre ellas (alrededor de 2,5 kilómetros entre cada una).

El fuero hacia especial hincapié -y ahi se puede ver la influencia de Olavide en su redactado- en el tamaño de las aldeas, que debian ser de 15,20, o 30 casas como máximo. Por tanto se trataba de impedir un crecimiento desmesurado. Aunque no se hacía la misma consideración para las feligresías, pensando que no llegarian a enfrentarse a un problema de desmesura. Se conocen los planos firmados por el ingeniero militar Simón Desnaux para Fuente Palmera y para una aldea genérica de esta feligresía, y pueden establecerse los límites, la forma y el tamaño de estas poblaciones: 16 casas en ambos casos. Seguramente los dos planos de 1768 son demostrativos del proyecto tal como se preveía ejecutar, cuando este ingeniero era director de Fuente Palmera y uno de los técnicos principales colaboradores del superintendente Olavide y del subdelegado de esta colonización de Andalucia que fue Fernando de Quintanilla.

\section{El fuero de colonización se}

promovió también con el fin de

establecer poblaciones en zonas

despobladas junto al nuevo

trazado del Camino Real de Cádiz

a Madrid, y así protegerlo

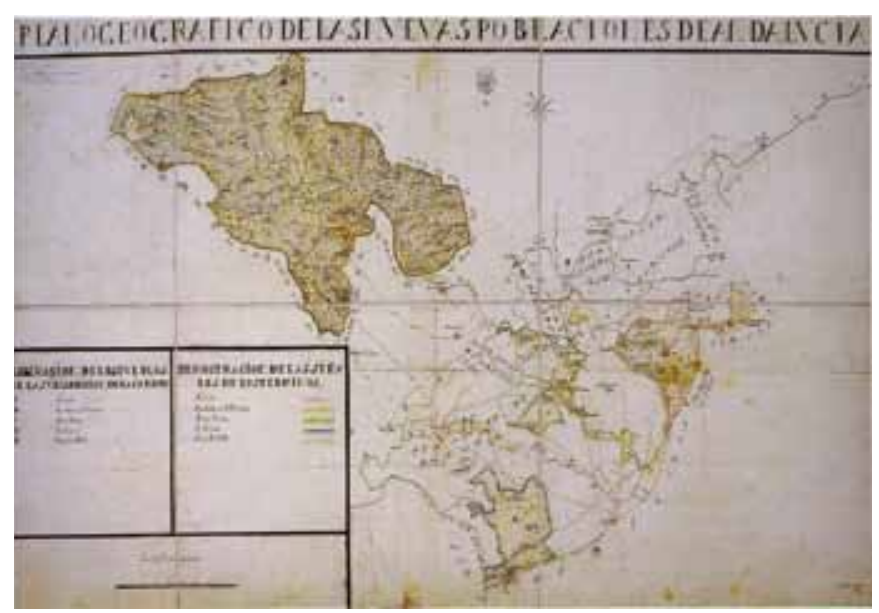

Plano Geográfico de las Nuevas Poblaciones de Andalucía (José de Ampudia y Valdés, 1794-1797). Fuente: Servicio Geográfico del Ejército

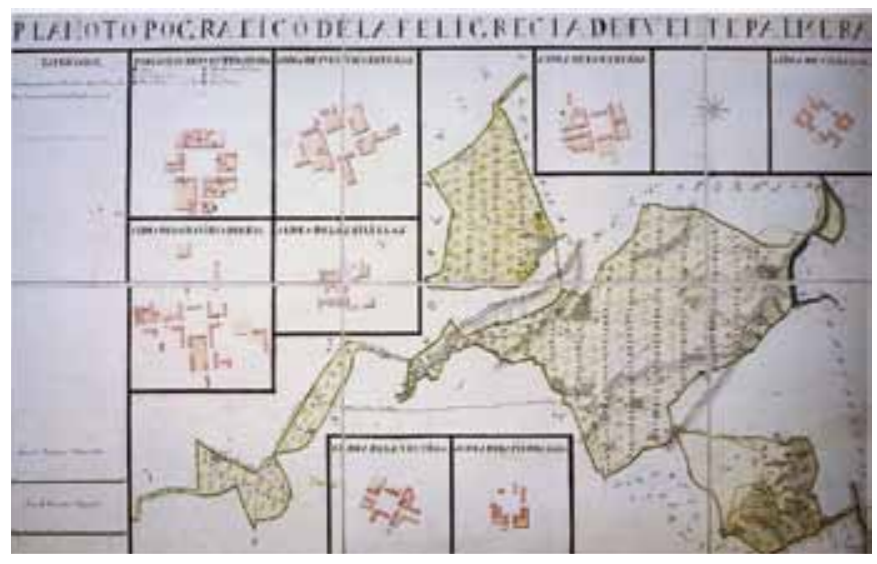

Plano Topográfico de la Feligresía de Fuente Palmera (José de Ampudia y Valdés, 1794-1797). Fuente: Servicio Geográfico del Ejército

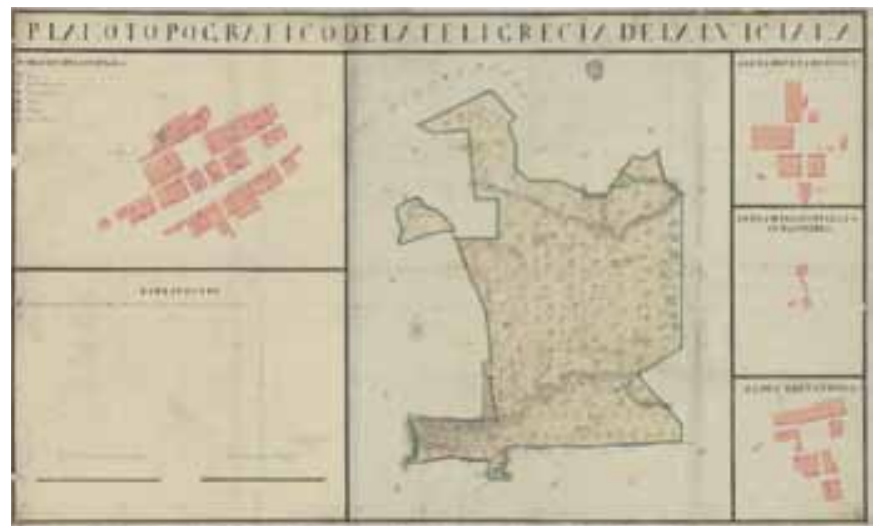

Plano Topográfico de la Feligresía de La Luisiana (José de Ampudia y Valdés, 1794-1797). Fuente: Archivo General Militar de Madrid (IHCM). SH.SE-1 1 / 2 
